\title{
Interacting roles of COMT and GAD1 genes in patients with treatment-resistant schizophrenia: A genetic association study of schizophrenia patients and healthy controls
}

\section{Masanobu Kogure}

Chiba University Graduate School of Medicine

Nobuhisa Kanahara ( $\nabla$ kanahara@faculty.chiba-u.jp )

Chiba University Center for Forensic Mental Health https://orcid.org/0000-0001-8550-8003

\section{Atsuhiro Miyazawa}

Chiba University Graduate School of Medicine

\section{Kengo Oishi}

Chiba University Graduate School of Medicine

\section{Yusuke Nakata}

Chiba University Graduate School of Medicine

\section{Yasunori Oda}

Chiba University Graduate School of Medicine

Masaomi lyo

Chiba University Graduate School of Medicine

\section{Research Article}

Keywords: clozapine, dopamine, GABA, single-nucleotide polymorphism, treatment-resistant

Posted Date: April 30th, 2021

DOI: https://doi.org/10.21203/rs.3.rs-462661/v1

License: (c) (1) This work is licensed under a Creative Commons Attribution 4.0 International License.

Read Full License

Version of Record: A version of this preprint was published at Journal of Molecular Neuroscience on June 14th, 2021. See the published version at https://doi.org/10.1007/s12031-021-01866-y. 


\section{Abstract \\ Background}

The projection from dopaminergic neurons to gamma-aminobutyric acid (GABA) interneurons in the prefrontal cortex is involved in the etiology of schizophrenia. The impact of interacting effects between dopamine signals and the expression of GABA on the clinical phenotypes of schizophrenia has not been studied. Since these interactions could be closely involved in prefrontal cortex functions, patients with specific alleles of these relevant molecules (which lead to lower or vulnerable genetic functions) may develop treatment-refractory symptoms. Subjects and

\section{Methods}

We conducted a genetic association study focusing on COMT and GAD1 genes for a treatment-resistant schizophrenia (TRS) group $(n=171)$, a non-TRS group $(n=592)$ and healthy controls $(H C: n=437)$, and we examined allelic combinations specific to TRS.

\section{Results}

The results revealed that the percentage of subjects with Met allele of rs 4680 on the COMT gene and C/C homozygote of rs3470934 of the GAD1 gene was significantly higher in the TRS group than the other two groups. There was no significant difference between the non-TRS group and HC groups.

\section{Conclusions}

Considering the direction of functions of these single-nucleotide polymorphisms revealed by previous studies, we speculate that subjects with the Met/CC allelic combination could have a higher dopamine level and a lower expression of GABA in the prefrontal cortex. Our results suggest that an interaction between the dopaminergic signal and GABA signal intensities could differ between TRS patients and patients with other types of schizophrenia and healthy subjects.

\section{Introduction}

It has been considered that in brains of patients with schizophrenia in the acute phase, the synthesis and release of dopamine are increased in the mesolimbic dopamine system, and it has been suggested that all antipsychotics exhibit preferable actions by blocking post-synaptic dopamine D2 receptors (DRD2) [Howes et al., 2009]. However, approx. $30 \%$ of patients with schizophrenia do not respond well to the standard antipsychotic medications despite sufficient usages in terms of both dose and duration, and these patients are considered to have treatment-resistant schizophrenia (TRS) [Elkis, 2007; Kane et al., 1988]. Patients with TRS generally continue to present with severe psychopathologies and experience an 
unstable clinical course for a long time period, leading to very poor outcomes. The clarification of the etiology of TRS and the development of a highly effective treatment for TRS thus remain important challenges in the field of psychiatry.

Schizophrenia patients have serious symptom domains that include negative symptoms and cognitive dysfunctions [Buchanan, 2007; Schaefer et al., 2013]. It is estimated that the neural networks involving these negative domains could differ from the networks underlying positive symptoms: that is, glutamatergic and $y$-aminobutyric acid (GABA) systems in the cortical area could be more directly involved in the origins of negative symptoms and cognitive dysfunctions [Schwartz et al., 2012; McCutcheon et al., 2020]. Several studies indicated that patients with TRS show more profound cognitive impairment compared to general (non-TRS) patients with schizophrenia [Joober et al., 2002; de Bartolomeis et al., 2013; Frydecka et al., 2016; Nakata et al., 2020]. Abnormalities in parvalbumin-positive GABA interneurons and lowered expressions of mRNA/protein of glutamic acid decarboxylase1 (GAD1) in the brains of individuals with schizophrenia have been consistently reported from multiple post-mortem studies, and these findings are suggested to be related to the lower expression of $y$-oscillation and cognitive dysfunctions in living patients with schizophrenia [Tallon-Baudry et al., 1998; Fries et al., 2001; Lewis et al., 2008; Buzsáki et al., 2004]. Clozapine is the only agent for which effectiveness against TRS has been established, and it is strongly suspected that GABA and glutamatergic neural systems are involved in the highly efficacious action of clozapine [O'Connor and O'Shea, 2015; Goldstein et al., 2015; Iwata et al., 2019].

In synapses in which dopaminergic neurons from the ventral tegmental area (VTA) innervate GABAergic interneurons in the prefrontal cortex (PFC), catechol-o-methyltransferase (COMT) plays an important role in the regulation of the dopamine signaling to GABAergic interneurons [Lewis et al., 2005; Lewis and Gonzalez-Burgos, 2006] because there are fewer dopamine transporters (another regulator of dopamine signal transduction) in the PFC region [Lewis et al., 2001; Kaenmaki et al., 2010]. Val158Met polymorphism (rs4680) on the COMT gene affects the activity of the COMT enzyme: Met homozygote shows $40 \%$ lower enzyme activity than Val homozygote, leading to an increase in the dopamine level in the PFC and anterior cingulate cortex (ACC) [Chen et al., 2004; Lachman et al., 1996]. Several studies reported that schizophrenia patients with Val/Val homozygote (presumable lower dopamine level) had lower densities of gray matter regions in the ACC, hippocampus, amygdala, and middle temporal cortex, and that these patients had severe negative symptoms and cognitive dysfunctions [Barnett et al., 2007; Tsuchimine et al., 2013; Apud and Weinberger, 2007]. In addition, numerous studies have indicated that the Val158Met polymorphism could be significantly related to cognitive functions in healthy subjects or patients with other psychiatric disorders other than schizophrenia [Nogueira et al., 2019; Pigoni et al., 2019].

Here, to examine the neural substrates specific to TRS (which could be different from those of other types of schizophrenia), we conducted a genetic association analysis focusing on dopamine-GABA interaction. Toward this goal, we selected rs4680 on the COMT gene and rs3749034 on the GAD1 gene, which were strongly suggested to affect the transcription of GAD1 protein [Straub et al., 2007; Tao et al., 2018]. 
Although there have been several analyses of interacting effects of COMT and GAD1 [Straub et al., 2007; Kirenskaya et al., 2018], their findings were not consistent. In the study by Straub et al. [2007], none of the single-nucleotide polymorphisms (SNPs) on the GAD1 gene were related to schizophrenia, but several SNPs created by combining COMT Val/Val homozygotes showed a significant relationship with vulnerability to schizophrenia. In a study by Kirenskaya et al. [2018], subjects with the Val/Val homozygote of COMT rs 4680 showed a longer response latency and poorer response rate in a saccade movement task. Whereas patients with $\mathrm{C} / \mathrm{C}$ homozygote of GAD1 rs3749034 exhibited a delayed response time, the T-allele-carrier patients showed a higher error rate on the task. Collectively, SNPs on the COMT and GAD1 genes could affect the disease vulnerability and cognitive impairments to some degree, but analyses of combined effects of the two genes have been insufficient. The present study is the first to analyze the interaction between COMT and GAD1 genes focusing on TRS patients. We hypothesize that TRS patients may have a more clearly allelic combination with a pair of alleles which are more vulnerable to schizophrenia.

\section{Subjects And Methods \\ 2.1. Subjects}

The case of a total of 763 patients were analyzed herein. All of them meet the criteria of schizophrenia or schizoaffective disorder in the Diagnostic and Statistical Manual of Mental Disorders, fourth edition, text revision (DSM- \-TR) or DSM-5, which were diagnosed by experienced psychiatrist $\$. Patients with any other psychiatric disorder as a comorbidity (other than nicotine dependence) were excluded. A total of 437 healthy subjects were also recruited as individuals in the healthy control $(\mathrm{HC})$ group; they were confirmed to have no past history or family member with any psychiatric or neurological disorder. All of the patients and controls were Japanese. The data of rs 4680 on COMT gene was partly overlapped with the previous two studies from our team [Oishi et al., 2018; 2020].

Blood drawing from each subject was performed after acquiring his/her written informed consent after a detailed explanation of the study. In the informed consent process, two psychiatrists judged the patient's ability to consent. If the patient was judged to be unable to understand the contents of the research due to his/her disease condition, the researchers were given informed consent from the patient's family member(s). This study was approved by the Ethics Committee of the Chiba University Graduate School of Medicine and was conducted following the Helsinki declaration.

\subsection{The diagnosis of treatment-resistant schizophrenia}

The diagnosis of having TRS for a schizophrenia patient was made based on both the assessment by the patient's physician(s) and all available medical records. The criteria of TRS were based on the Clozapine Patients Monitoring Service (CPMS) criteria, and the present study included only the non-responder type of schizophrenia patient: this type was defined as never having shown a sufficient response (i.e., $<40$ points on Global Assessment of Functioning over the past 12 months) to at least two types of 
antipsychotics with each chlorpromazine-equivalent dose of $>600 \mathrm{mg}$ for $>4$ weeks. Patients who were judged as belonging to the intolerance type were excluded from this study.

\subsection{Genotyping SNPs}

The genome was derived from each subject's blood sample with the QIAamp DNA Blood Mini Kit (250) (Qiagen, Valencia, CA). We examined two SNPs: rs4680 (Val158Met: $\mathrm{G}>\mathrm{A}$ ) in exon3 of COMT gene and rs3749034(C > T) in the 5'-UTR of GAD1 gene. The genotypes of these SNPs were determined by a TaqMan probe assay (Applied Biosystems, Foster City, CA) with an ABI PRISM 7300 real-time polymerase chain reaction (PCR) system (Applied Biosystems). All procedures followed the manufacturer's manual: one cycle of $95^{\circ} \mathrm{C}$ for 10 mins followed by 40 cycles of $92^{\circ} \mathrm{C}$ for 15 secs and $60^{\circ} \mathrm{C}$ for 60 secs.

\subsection{Statistical analyses}

All statistical procedures were performed with SPSS ver. 19.0 software (IBM, NY). For the subjects' demographic information, we performed an analysis of variance (ANOVA) and used the $\chi^{2}$-test to the continuous variable (age) and categorical variable (sex), respectively. As a post-hoc test, Bonferroni correction was then conducted for the ANOVA and a residual analysis was conducted for the $\chi^{2}$-test.

Previous investigations of Japanese subjects conducted by other teams indicated that the allelic distributions of both rs 4680 and rs3749034 were not significantly different between subjects with schizophrenia and healthy subjects. When we calculated the necessary sample sizes to reach statistical significance in the present comparison of the schizophrenia groups and healthy controls in reference to the two prior studies with the largest Japanese sample sizes to date [lkeda et al., 2007; Okochi et al., 2009], the value for rs 4680 was $n=121,410$ per group and that for rs3749034 was $n=23,908$ per group under the condition of $\alpha=0.05, \beta=0.20$, and power $=0.80$. The present study's sample size was much smaller than these estimations, and we therefore set the statistical significance level at $p<0.10$ in the

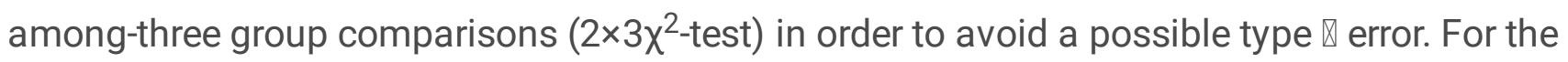
identification of the cell(s) with a significant increase or decrease, we performed the residual analysis with the statistical significance set at $p<0.05$.

\section{Results}

As shown in Table 1, the dominant gender was opposite between the Non-TRS and HC groups, and the gender distribution in the TRS group was approximately equal. The age at blood sample drawing also differed among the three groups: TRS > Non-TRS > HC group. 
Table 1

Demographic information of the treatment-resistant schizophrenia (TRS), Non-TRS, and healthy control (HC) groups

\begin{tabular}{|c|c|c|c|c|c|}
\hline \multirow[t]{2}{*}{ Variables } & \multirow{2}{*}{$\begin{array}{l}\text { TRS } \\
\text { group } \\
n=171\end{array}$} & \multirow{2}{*}{$\begin{array}{l}\text { Non-TRS } \\
\text { group } \\
n=592\end{array}$} & \multirow{2}{*}{$\begin{array}{l}\text { HC group } \\
n=437\end{array}$} & \multicolumn{2}{|l|}{ Statistics } \\
\hline & & & & Values & Post-hoc \\
\hline $\begin{array}{l}\text { Sex: } \\
\text { male/female, } \\
\text { n }\end{array}$ & $86 / 85$ & $280 / 310 *$ & $248 / 185^{\star}$ & $\begin{array}{l}\chi^{2}=9.737, p \\
=0.008\end{array}$ & $\begin{array}{l}\text { Male, NonTRS }<\text { HC; Female, } \\
\text { NonTRS }>\text { HC }\end{array}$ \\
\hline Age, yrs $\#$ & $\begin{array}{l}49.65 \\
{[13.04]}\end{array}$ & $\begin{array}{l}45.03 \\
{[15.35]^{\dagger}}\end{array}$ & $\begin{array}{l}36.36 \\
{[15.06]^{\dagger}}\end{array}$ & $\begin{array}{l}F=64.81, \mathrm{p}< \\
0.001\end{array}$ & TRS $>$ NonTRS $>\mathrm{HC}$ \\
\hline \multicolumn{6}{|c|}{${ }^{\mathrm{D}}$ The sex of two subjects in the Non-TRS group and four in the HC group were unknown. } \\
\hline
\end{tabular}

The two above-described SNPs did not deviate from Hardy-Weinberg equilibrium in either schizophrenia group or the HC group. For rs4680, no significant difference was observed among the three groups ( $p>$ 0.10). For rs3759034, however, there was a significant difference among the three groups: $\mathrm{C} / \mathrm{C}$ homozygote was significantly dominant in the TRS group whereas T-allele carriers was the dominant type in the HC group $\left(\chi^{2}=4.775, p=0.092\right.$; Table 2$)$. However, the residual analysis as a post-hoc test did not show any cell reaching statistical significance $(p>0.05)$.

Table 2

Genotype distributions of rs4680 on COMT gene and of rs3749034 on GAD1 gene
TRS group
Non-TRS group
$\mathrm{HC}$ group
MAF $^{\# \quad \text { Statistic values }}$

rs4680

$\begin{array}{lllll}\text { Met (-) } & 75(43.4 \%) & 274(46.4 \%) & 192(43.9 \%) & 45.4 \%\end{array} \quad \chi^{2}=0.725, p=0.696$

rs3749034

$\begin{array}{lllll}\text { T (-) } & 95(55.6 \%) & 291(49.2 \%) & 200(45.8 \%) & 45.2 \%\end{array} \chi^{2}=4.775, \mathrm{p}=0.092$

\# MAF data were derived from healthy subjects in Kochi et al. [2009] for rs4680 and Ikeda et al. [2007] for rs3749034.

Based on several previous studies, we divided the genotypes of rs4680 into three subgroups (Val/Val, $\mathrm{Val} / \mathrm{Met}$, and Met/Met) and divided the genotypes of rs3749034 into two subgroups (C/C and C/T + T/T), taking the number of each genotype into consideration (Table 3 ). When the combinations of both SNPs 
were compared among the three diagnosis groups, there was a significant difference $\left(\chi^{2}=5.050, p=\right.$ $0.080)$. The residual analysis indicated that the TRS group had a higher percentage of subjects with Met(+) + T(-) compared to the other two groups, whereas the percentage of subjects in the TRS group with other allelic combinations was lower than those in the other two groups.

Table 3

Combined analysis of genotypes of rs4680 on COMT gene and rs3749034 on GAD1 gene

\begin{tabular}{|c|c|c|c|c|}
\hline rs4680/rs3749034 & TRS group & Non-TRS group & HC group & Statistic values \\
\hline $\operatorname{Met}(+) / T(-)$ & $56(32.7 \%)$ & $148(25.0 \%)$ & $106(24.3 \%)$ & \multirow{2}{*}{$\chi^{2}=5.050, p=0.080$} \\
\hline Others & $115(67.3 \%)$ & $444(75.0 \%)$ & 331 (75.7\%) & \\
\hline
\end{tabular}

\section{Discussion}

Accumulating evidence from multiple studies suggests that the brains of patients with schizophrenia have impaired GABA functions. GAD1 plays a main role in the synthesis of GABA in the central nervous system (CNS), accounting for approx. $80 \%$ of the GABA production in the CNS (the remaining $20 \%$ of GABA is produced by GAD2) [Asada et al., 1997; Condie et al., 1997;Mitchell et al., 2015『Varju et al., 2002]. The synthesis of GABA is influenced by SNPs on the GAD1 gene [Straub et al., 2007; Marenco et al., 2010; Tao et al., 2018] and regulation by a dopamine signal [Shukla et al., 2016]. In the present study we examined genetic influences of the functional SNPs (rs4680 and rs3749034) on the severity of schizophrenia, and the results revealed that the TRS group included a greater percentage of patients with Met carrier of rs4680 (lower COMT enzyme, leading to higher dopamine in the PFC) and T-allele noncarriers (i.e., C/C homozygote) of rs3749034 compared to the Non-TRS group and HC group. There was no significant difference in the allelic distribution between the Non-TRS and HC groups.

This finding might be somewhat unexpected since Met allele innervates a higher dopamine level in the PFC. We also observed that C-allele, the major allele of rs3749034, was significantly dominant in the TRS group by its single analysis with a less rigorous statistical threshold, and this allele appears to be less related to the vulnerability to schizophrenia.

Regarding COMT gene, several studies have suggested that this gene could be related to treatmentrefractoriness or responsiveness to medications, and most of those studies demonstrated that Met-allele carriers could be linked to treatment-refractoriness [Inada et al., 2003; Sagud et al., 2018; Hajj et al., 2019] with the exception of one report [Terzić et al., 2016], which was in line with our present finding. However, some research groups examining COMT and GAD1 suggested that the interacting effects of both genes could not simply explain the synthesis of GABA and the clinical phenotypes in schizophrenia. For example, in a magnetic resonance spectroscopy (MRS) study of healthy individuals by Marenco et al. [2010], the subjects with the Val/Met heterozygote of COMT rs4680 showed higher GABA concentrations compared to those with other homozygotes (i.e., Val/Val and Met/Met homozygotes). In that study, the subjects with minor alleles of two SNPs (rs1978340 and rs769390, which were not examined in our 
present study) among three SNPs that significantly affect the GABA concentration showed higher GABA levels compared to those with major alleles of these polymorphisms. A study by Straub et al. [2007] demonstrated that rs3749034 of GAD1 was the only polymorphism affecting the expression of GAD1 mRNA and that its major allele (i.e., G allele) was related to lower expression. In a total of 19 SNPs on the GAD1 gene examined in that study, no significant SNP was observed to be significantly related to schizophrenia, but when the interactions between these SNPs and rs4680 Val/Val homozygote were examined, there were some SNPs relate to the disease vulnerability: these relevant SNPs were a mixture of major and minor alleles, and it is thus difficult to estimate the impacts of these SNPs on the transcription of GAD1. Recent evidence demonstrated that GABA synthesis could be influenced by the methylation of GAD1 [Tao et al., 2018], suggesting that such other factors should be taken into consideration concerning the production of GABA.

The projection of dopamine signal to the GABAergic neurons in the PFC is modulated by both excitatory dopamine D1 receptors (DRD1) and inhibitory DRD2. Pyramidal neurons are also involved in this modulation [Momiyama and Nishijo, 2017; Shukla et al., 2016]. It has been long believed that higher or lower dopamine signal transduction had negative influences on PFC-modulating cognitive functions, and that this characteristic show a so-called inverted U-shape curve [Lidow et al., 1998; Ira et al., 2013]. That is, the dopamine network projects onto both GABAergic interneurons and glutamatergic pyramidal neurons, which further project to other pyramidal neurons: these are connected to each other in a very complicated manner, and they determine the excitatory and inhibitory balance in the PFC [Sohal and Rubenstein, 2019]. According to a post-mortem study, healthy individuals with COMTVal/Val homozygote (which may lower the dopamine level) showed higher mRNA of GAD1 compared to individuals with Met/Met homozygote [Shukla et al., 2016], suggesting the maintenance of the homeostasis of signal transmissions onto the PFC in healthy individuals. In contrast, subjects with schizophrenia had GAD1 mRNA levels that did not differ between those with Val/Val and those with Met/Met homozygotes, possibly indicating an attenuated ability to maintain homeostasis. These findings suggest the possibility that schizophrenia patients have a weakened capacity to adjust to a variety of stimulations from the external world, further leading to the origins of cognitive impairments at the cortical level and impaired dopamine synthesis at the subcortical level.

In the present study, when we divided the schizophrenia patients into the TRS and Non-TRS groups, we observed that these groups' SNP distributions of COMT and GAD1 differed: some of the TRS patients had a combination of the two genes that differed from those of non-TRS patients or healthy subjects, and we suspect that this may provide a clue to the knowledge of the interacting roles of the two molecules in the disease etiology. Recent studies of TRS patients indicated that the synthesis of dopamine or the dopamine volume in the synaptic clefts could differ in patients with TRS compared to other schizophrenia patients who achieve a good response to antipsychotic medications [Demjaha et al., 2012; Kim et al., 2017]. Several studies uniformly observed that more severe cognitive impairments in TRS patients [Joober et al., 2002; de Bartolomeis et al., 2013; Frydecka et al., 2016; Nakata et al., 2020], and another line of evidence suggests that such severe cognitive dysfunctions could be related to an attenuation of GABA signal transduction [Tallon-Baudry et al., 1998; Fries et al., 2001; Lewis et al., 2008; 
Buzsáki et al., 2004]. It was also reported that clozapine could enhance GABA signal transduction [Daskalalis et al., 2008; Liu et al., 2009; Kaster et al., 2015; Miyazawa et al., 2021]. Collectively, these findings suggest that TRS patients could have a neural substrate that is distinct from those of other general schizophrenia patients. The present results may indicate that a specific allele combination of $C O M T$ and $G A D 1$ genes provides consistently lower GABA systems, leading to treatment-refractory symptoms. It is important to study the relationships among SNPs of COMT/GAD1 and GABA concentrations in the human brain by MRS techniques.

The present study was preliminary, with several weaknesses as follows. The sample sizes of both the patients and controls were too small to reach a firm conclusion. We did not examine the cognitive functions of the subjects. Although most of the prior relevant studies seemed to presume that the interaction of dopamine and GABA in the PFC underlies cognitive impairments in schizophrenia, we did not directly examine cognitive functions of our participants. However, this study has some strengths: the TRS patients were well characterized and selected through a process that was identical to clinical practice when the introduction of clozapine is considered. Another strength is that the two SNPs examined are well characterized in terms of their function and interaction, providing the opportunity to investigate the patients' brain network from a genetic perspective.

In conclusion, our findings are preliminary, but by focusing on TRS patients, the data suggest that this group could have one or more different COMT and GAD1 allelic combinations compared to non-TRS patients or healthy controls. Overall, our results suggest that TRS patients are less capable of appropriately tuning the excitatory and inhibitory balance and homeostasis, leading to a difficulty in regulating the subcortical dopamine system and to severe cognitive impairments and negative symptoms.

\section{Declarations}

\section{Acknowledgements}

None.

\section{References}

Apud JA, Weinberger DR (2007) Treatment of cognitive deficits associated with schizophrenia: potential role of catechol-0-methyltransferase inhibitors. CNS Drugs 21: 535-557.

Asada H, Kawamura Y, Maruyama K, Kume H, Ding RG, Kanbara N, Kuzume H, Sanbo M, Yagi T, Obata K (1997) Cleft palate and decreased brain gamma-aminobutyric acid in mice lacking the 67-kDa isoform of glutamic acid decarboxylase. Proc Natl Acad Sci U S A 94: 6496-6499.

Barnett JH, Jones PB, Robbins TW, Müller U (2007) Effects of the catechol-O-methyltransferase Val158Met polymorphism on executive function: a meta-analysis of the Wisconsin Card Sort Test in 
schizophrenia and healthy controls. Mol Psychiatry 12: 502-509.

Buchanan RW (2007) Persistent negative symptoms in schizophrenia: an overview. Schizophr Bull 33: 1013-1022.

Buzsáki G, Geisler C, Henze DA, Wang XJ (2004) Interneuron Diversity series: Circuit complexity and axon wiring economy of cortical interneurons. Trends Neurosci 27:186-193.

Chen J, Lipska BK, Halim N, Ma QD, Matsumoto M, Melhem S, Kolachana BS, Hyde TM, Herman MM, Apud J, Egan MF, Kleinman JE, Weinberger DR (2004) Functional analysis of genetic variation in catechol-0-methyltransferase (COMT): effects on mRNA, protein, and enzyme activity in postmortem human brain. Am J Hum Genet 75: 807-821.

Condie BG, Bain G, Gottlieb DI, Capecchi MR (1997) Cleft palate in mice with a targeted mutation in the gamma-aminobutyric acid-producing enzyme glutamic acid decarboxylase 67. Proc Natl Acad Sci U S A 94: 11451-11455.

de Bartolomeis A, Prinzivalli E, Callovini P, D'Ambrosio L, Altavilla B, Avagliano C, lasevoli F (2018) Treatment resistant schizophrenia and neurological soft signs may converge on the same pathology: Evidence from explanatory analysis on clinical, psychopathological and cognitive variables. Progress in Neuropsychopharmacology and Biological Psychiatry 81: 356-366.

Daskalakis ZJ, Christensen BK, Fitzgerald PB, Moller B, Fountain SI, Chen R (2008) Increased cortical inhibition in persons with schizophrenia treated with clozapine. J Psychopharmacol 22: 203-209.

Demjaha A, Murray RM, McGuire PK, Kapur S, Howes OD (2012) Dopamine synthesis capacity in patients with treatment-resistant schizophrenia. Am J Psychiatry 169:1203-1210.

Elkis H (2007) Treatment-resistant schizophrenia. Psychiatr Clin North Am 30: 511-533. Kane J, Honigfeld G, Singer J, Meltzer H (1988) Clozapine for the treatment-resistant schizophrenic. A double-blind comparison with chlorpromazine. Arch Gen Psychiatry 45: 789-796.

Fries P, Neuenschwander S, Engel AK, Goebel R, Singer W (2001) Rapid feature selective neuronal synchronization through correlated latency shifting. Nat Neurosci 4: 194-200.

Frydecka D, Beszłej JA, Gościmski P, Kiejna A, Misiak B (2016) Profiling cognitive impairment in treatment-resistant schizophrenia patients. Psychiatry Research 235: 133-138.

Goldstein ME, Anderson VM, Pillai A, Kydd RR, Russell BR (2015) Glutamatergic neurometabolites in clozapine-responsive and -resistant schizophrenia. Int J Neuropsychopharmacol 18:pyu117.

Hajj A, Obeid S, Sahyoun S, Haddad C, Azar J, Rabbaa Khabbaz L, Hallit S (2019) Clinical and Genetic Factors Associated with Resistance to Treatment in Patients with Schizophrenia: A Case-Control Study. Int J Mol Sci 20: 4753. 
Howes OD, Kapur S (2009) The dopamine hypothesis of schizophrenia: version III-the final common pathway. Schizophr Bull 35: 549-562.

Ikeda M, Ozaki N, Yamanouchi Y, Suzuki T, Kitajima T, Kinoshita Y, Inada T, Iwata N (2007) No association between the glutamate decarboxylase 67 gene (GAD1) and schizophrenia in the Japanese population. Schizophr Res 91: 22-26.

Inada T, Nakamura A, lijima Y (2003) Relationship between catechol-O-methyltransferase polymorphism and treatment-resistant schizophrenia. Am J Med Genet B Neuropsychiatr Genet 120B(1): 35-39.

Iwata Y, Nakajima S, Plitman E, Caravaggio F, Kim J, Shah P, Mar W, Chavez S, De Luca V, Mimura M, Remington G, Gerretsen P, Graff-Guerrero A (2019) Glutamatergic Neurometabolite Levels in Patients With Ultra-Treatment-Resistant Schizophrenia: A Cross-Sectional 3T Proton Magnetic Resonance Spectroscopy Study. Biol Psychiatry 85: 596-605.

Joober R, Rouleau GA, Lal S, Dixon M, O'Driscoll G, Palmour R, Annable L, Bloom D, Lalonde P, Labelle A, Benkelfat C (2002) Neuropsychological impairments in neuroleptic-responder vs. -nonresponder schizophrenic patients and healthy volunteers. Schizophrenia Research 53: 229-238.

Käenmäki M, Tammimäki A, Myöhänen T, Pakarinen K, Amberg C, Karayiorgou M, Gogos JA, Männistö PT (2010) Quantitative role of COMT in dopamine clearance in the prefrontal cortex of freely moving mice. $J$ Neurochem 114: 1745-1755.

Kaster TS, de Jesus D, Radhu N, Farzan F, Blumberger DM, Rajji TK, Fitzgerald FB, Daskalakis ZJ (2015) Clozapine potentiation of GABA mediated cortical inhibition in treatment resistant schizophrenia. Schizophr Res 165: 157-162.

Kim E, Howes OD, Veronese M, Beck K, Seo S, Park JW, Lee JS, Lee YS, Kwon JS (2017) Presynaptic Dopamine Capacity in Patients with Treatment-Resistant Schizophrenia Taking Clozapine: An [(18)F]DOPA PET Study. Neuropsychopharmacology 42: 941-950.

Kirenskaya AV, Storozheva ZI, Gruden MA, Sewell RDE (2018) COMT and GAD1 gene polymorphisms are associated with impaired antisaccade task performance in schizophrenic patients. Eur Arch Psychiatry Clin Neurosci 268: 571-584.

Lachman HM, Papolos DF, Saito T, Yu YM, Szumlanski CL, Weinshilboum RM (1996) Human catechol-Omethyltransferase pharmacogenetics: description of a functional polymorphism and its potential application to neuropsychiatric disorders. Pharmacogenetics 6: 243-250.

Lewis DA, Melchitzky DS, Sesack SR, Whitehead RE, Auh S, Sampson A (2001) Dopamine transporter immunoreactivity in monkey cerebral cortex: regional, laminar, and ultrastructural localization. J Comp Neurol 432: 119-136.

Lewis DA, Hashimoto T, Volk DW (2005) Cortical inhibitory neurons and schizophrenia. 
Nat Rev Neurosci 6: 312-324.

Lewis DA, Gonzalez-Burgos G (2006) Pathophysiologically based treatment interventions in schizophrenia. Nat Med 12:1016-1022.

Lewis DA, Cho RY, Carter CS, Eklund K, Forster S, Kelly MA, Montrose D (2008) Subunit-selective modulation of GABA type A receptor neurotransmission and cognition in schizophrenia. Am J Psychiatry 165: 1585-1593.

Lidow MS, Williams GV, Goldman-Rakic PS (1998) The cerebral cortex: a case for a common site of action of antipsychotics. Trends Pharmacol Sci 19: 136-140.

Liu SK, Fitzgerald PB, Daigle M, Chen R, Daskalakis ZJ (2009) The relationship between cortical inhibition, antipsychotic treatment, and the symptoms of schizophrenia. Biol Psychiatry 65: 503-509.

Marenco S, Savostyanova AA, van der Veen JW, Geramita M, Stern A, Barnett AS, Kolachana B, Radulescu E, Zhang F, Callicott JH, Straub RE, Shen J, Weinberger DR (2010) Genetic modulation of GABA levels in the anterior cingulate cortex by GAD1 and COMT. Neuropsychopharmacology 35: 1708-1717.

McCutcheon RA, Krystal JH, Howes OD (2020) Dopamine and glutamate in schizophrenia: biology, symptoms and treatment. World Psychiatry 19: 15-33.

Mitchell AC, Jiang Y, Peter C, Akbarian S (2015) Transcriptional regulation of GAD1 GABA synthesis gene in the prefrontal cortex of subjects with schizophrenia. Schizophr Res 167: 28-34.

Miyazawa A, Kanahara K, Nakata Y, Kodama S, Kimura H, Kimura A, Oda Y, Watanabe H, lyo M (2021) Clozapine prolongs cortical silent period in patients with treatment-resistant schizophrenia.

Psychophrmacol Bull In Press.

Momiyama T, Nishijo T (2017) Dopamine and Serotonin-Induced Modulation of GABAergic and Glutamatergic Transmission in the Striatum and Basal Forebrain. Front Neuroanat 11: 42.

Nakata Y, Kanahara N, Kimura A, Niitsu T, Komatsu H, Oda Y, Ishikawa M, Hasegawa T, Kamata Y, Yamauchi A, Inazumi K, Kimura H, lyo M (2020) Autistic traits and cognitive profiles of treatment-resistant schizophrenia. Schizophr Res Cogn 22:100186.

Nogueira NGHM, Bacelar MFB, Ferreira BP, Parma JO, Lage GM (2019) Association between the catecholO-methyltransferase (COMT) Val158Met polymorphism and motor behavior in healthy adults: A study review. Brain Res Bull 144: 223-232.

O'Connor WT, O'Shea SD (2015) Clozapine and GABA transmission in schizophrenia disease models: establishing principles to guide treatments. Pharmacol Ther 150: 47-80. 
Oishi K, Kanahara N, Takase M, Oda Y, Nakata Y, Niitsu T, Ishikawa M, Sato Y, lyo M (2018) Vulnerable combinations of functional dopaminergic polymorphisms to late-onset treatment resistant schizophrneia. Plos One 13: e0207133.

Oishi K, Niitsu T, Kanahara N, Hashimoto T, Komatsu H, Sasaki T, Takase M, Sato Y, lyo M (2020) Genetic combination risk for schizophrenia. Schizophr Research 215: 473-474.

Okochi T, Ikeda M, Kishi T, Kawashima K, Kinoshita Y, Kitajima T, Yamanouchi Y, Tomita M, Inada T, Ozaki $\mathrm{N}$, Iwata N (2009) Meta-analysis of association between genetic variants in COMT and schizophrenia: an update. Schizophr Res 110: 140-148.

Pigoni A, Lazzaretti M, Mandolini GM, Delvecchio G, Altamura AC, Soares JC, Brambilla P (2019) The impact of COMT polymorphisms on cognition in Bipolar Disorder: A review. J Affect Dis 243; 545-551.

Sagud M, Tudor L, Uzun S, Perkovic MN, Zivkovic M, Konjevod M, Kozumplik O, Cusa BV, Strac DS, Rados I, Mimica N, Peles AM, Erjavec GN, Pivac N (2018) Haplotypic and Genotypic Association of Catechol- OMethyltransferase rs 4680 and rs4818 Polymorphisms and Treatment Resistance in Schizophrenia. Front Pharmacol 9: 705.

Schaefer J, Giangrande E, Weinberger DR, Dickinson D (2013) The global cognitive impairment in schizophrenia: consistent over decades and around the world. Schizophr Res 150: 42-50.

Schwartz TL, Sachdeva S, Stahl SM (2012) Glutamate neurocircuitry: theoretical underpinnings in schizophrenia. Front Pharmacol 3: 195.

Shukla AA, Jha M, Birchfield T, Mukherjee S, Gleason K, Abdisalaam S, Asaithamby A, Adams-Huet B, Tamminga CA, Ghose S (2016) COMT val158met polymorphism and molecular alterations in the human dorsolateral prefrontal cortex: Differences in controls and in schizophrenia. Schizophr Res 173: 94-100.

Sohal VS, Rubenstein JLR (2019) Excitation-inhibition balance as a framework for investigating mechanisms in neuropsychiatric disorders. Mol Psychiatry 24: 1248-1257.

Straub RE, Lipska BK, Egan MF, Goldberg TE, Callicott JH, Mayhew MB, Vakkalanka RK, Kolachana BS, Kleinman JE, Weinberger DR (2007) Allelic variation in GAD1 (GAD67) is associated with schizophrenia and influences cortical function and gene expression. Mol Psychiatry 12: 854-869.

Tallon-Baudry C, Bertrand O, Peronnet F, Pernier J (1998) Induced gamma-band activity during the delay of a visual short-term memory task in humans. J Neurosci 18: 4244-4254.

Tao R, Davis KN, Li C, Shin JH, Gao Y, Jaffe AE, Gondré-Lewis MC, Weinberger DR, Kleinman JE, Hyde TM (2018) GAD1 alternative transcripts and DNA methylation in human prefrontal cortex and hippocampus in brain development, schizophrenia. Mol Psychiatry 23: 1496-1505. 
Terzić T, Kastelic M, Dolžan V, Plesničar BK (2016) Genetic polymorphisms in dopaminergic system and treatment-resistant schizophrenia. Psychiatr Danub 28: 127-131.

Tsuchimine S, Yasui-Furukori N, Kaneda A, Kaneko S (2013) Differential effects of the catechol-Omethyltransferase Val158Met genotype on the cognitive function of schizophrenia patients and healthy Japanese individuals. PLoS One 8: e76763.

Varju P, Katarova Z, Madarász E, Szabó G (2002) Sequential induction of embryonic and adult forms of glutamic acid decarboxylase during in vitro-induced neurogenesis in cloned neuroectodermal cell-line, NE7C2. J Neurochem 80: 605-615.

Williams GV, Castner SA (2006) Under the curve: critical issues for elucidating D1 receptor function in working memory. Neuroscience. 139: 263-276. 